FNAL-PUB/97-XXX

CERN-TH/97-373

hep-ph/9801251

\title{
Photon signatures for low energy supersymmetry breaking and broken R-parity
}

\author{
M. Carena ${ }^{a}$, S. Pokorski ${ }^{b, c}$ and C.E.M. Wagner ${ }^{b}$ \\ ${ }^{a}$ Fermi National Accelerator Laboratory, P.O. Box 500, Batavia, IL 60510, U.S.A. \\ ${ }^{b}$ CERN, TH Division, CH-1211 Geneva 23, Switzerland \\ ${ }^{c}$ Institute of Theoretical Physics, Warsaw University, Poland.
}

\begin{abstract}
The possible phenomenological consequences of R-parity violating interactions in the framework of low energy supersymmetry breaking are studied. It is pointed out that even very weak R-parity violation would completely overshadow one of the basic signatures of low energy supersymmetry breaking models, that is the decay of the next to lightest supersymmetric particle into a photon (lepton) and missing energy. Thus, the observation of these decays would put very strong limits on R-parity violating couplings. Viceversa, if R-parity violation is established experimentally, before a detailed knowledge of the spectrum is obtained, it will be very difficult to distinguish gravity mediated from low energy gauge mediated supersymmetry breaking scenarios. Those conclusions are very model independent. We also comment on the possibility of mixing between charged and neutral leptons with charginos and neutralinos, respectively, and its phenomenological consequences for the photon (lepton) signatures, in scenarios where this mixing is generated by the presence of bilinear or trilinear R-parity violating terms in the superpotential.
\end{abstract}

CERN-TH/97-373

December 1997 
There are two basic open questions in supersymmetric models: The mechanism of supersymmetry (SUSY) breaking and the theory of flavor. The latter includes in particular the question about R-parity conservation or the pattern of its breaking. The two questions may or may not be correlated. In models in which supersymmetry breaking is transmited to the observable sector via gauge interactions at energies much lower than the grand unification scale, the flavor physics is most likely decoupled from the mechanism of supersymmetry breaking, due to a large separation in the relevant mass scales. The gauge mediation of SUSY breaking and R-parity violation is certainly one of the possible scenarios. The signatures for both, low energy gauge mediated supersymmetry breaking and R-parity violation are based on rare decays and it is hence of great importance to determine if both ideas can be independently established experimentally.

A quite general signature for low energy gauge mediated scenario of supersymmetry breaking is the decay of the next to lightest supersymmetric particle (NLSP) into gravitinos (the actual lightest supersymmetric particle, in this case) [四],

$$
\tilde{\chi}_{1}^{0} \rightarrow \gamma \tilde{G} ; \quad \text { or } \quad \tilde{l} \rightarrow l \tilde{G},
$$

depending on which sparticle is the NLSP.7 The decay rate, Eq(1), is given by [2]-[4],

$$
\Gamma \simeq K \frac{m_{N L S P}}{48 \pi}\left(\frac{m_{N L S P}}{\sqrt{M_{P} m_{\tilde{G}}}}\right)^{4}
$$

where $M_{P}$ is the Planck mass, $m_{N L S P}$ is the mass of the NLSP and $K$ is a projection factor equal to the square of the component in the NLSP of the superpartner of the particle the NLSP is decaying into. For instance, if the NLSP is a neutralino, which decays into a photon and a gravitino, $K$ is just the square of the photino component of the lightest neutralino, which is given by

$$
K=\left|N_{11} \cos \theta_{W}+N_{12} \sin \theta_{W}\right|^{2}
$$

where $N_{i j}$ is the mixing matrix connecting the neutralino mass eigenstates to the weak eigenstates in the basis $\tilde{B}, \tilde{W}, \tilde{H}_{1}, \tilde{H}_{2}$. The decay rate is inversely proportional to the square of the gravitino mass, which is given by

$$
m_{\tilde{G}} M_{P} \simeq F_{S U S Y} / \sqrt{3}
$$

where $\sqrt{F_{\text {SUSY }}}$ is the supersymmetry breaking scale.

For our purpose, most interesting are the limits on $F_{S U S Y}$ coming from the requirement of detectability, this means, that the NLSP decays before escaping the detector. From Eq. (2), an upper bound on the supersymmetry breaking scale, $\sqrt{F_{\text {SUSY }}} \lesssim 10^{7}$ $\mathrm{GeV}$, may be obtained from the requirement that the NLSP decays into gravitinos within the detector [4]. Low energy SUSY breaking scenarios, with observable decays

\footnotetext{
${ }^{1}$ If the $\tilde{\chi}_{1}^{0}$ is mainly Higgsino- like, then the decay $\tilde{\chi}_{1}^{0} \rightarrow h \tilde{G} \rightarrow b \bar{b} \tilde{G}$, where $h$ is the lightest Higgs boson (but can also be the heavy CP-even Higgs or the CP-odd Higgs boson if they are sufficiently light), is also possible.
} 
into photon and gravitino will be characterized by events containing photons and missing energy, in contrast to supergravity scenarios, where, unless very specific conditions are fulfilled [5], [6], photons do not represent a characteristic signature.

Renormalizable R-parity violating scenarios are well known [7]. They are derived from the effective low-energy superpotential

$$
W=\lambda_{i j k} L_{i} L_{j} \bar{E}_{k}+\lambda_{i j k}^{\prime} L_{i} Q_{j} \bar{D}_{k}+\lambda_{i j k}^{\prime \prime} \bar{U}_{i} \bar{D}_{j} \bar{D}_{k}+\epsilon_{i} L_{i} H_{2}
$$

where $L(E)$ are the left- (right-) handed lepton $S U(2)_{L}$ doublet (singlet) superfields, $Q$ are the left handed quark doublet superfields, and $U$ and $D$ are the up and down right-handed quark superfields. If present, R-parity violating couplings may induce a large number of new lepton or baryon number violating processes. Hence, strong experimental limits on many of the R-parity violating couplings, or on their products, can be set [9]. We discuss trilinear and bilinear terms independently and begin with the former.

One obvious, but important, observation is that, in the case of a neutralino as the NLSP, the presence of any single trilinear R-parity violating coupling would induce the decay

$$
\tilde{\chi}_{1}^{0} \rightarrow f f f
$$

In the case of lepton violating couplings and sleptons as the NLSP,

$$
\tilde{l} \rightarrow f f
$$

where $f$ are fermionic states, which may be leptonic or hadronic depending on the particular cases. The rate for the decay of neutralino into fermions via R-parity violating interactions reads [7], 8]

$$
\Gamma\left(\tilde{\chi}_{1}^{0} \rightarrow f f f\right) \simeq \frac{\lambda^{2} \alpha_{w}}{128 \pi^{3}}\left[N_{11} \tan \theta_{w} \frac{Y_{f}}{2}+N_{12} T_{f}^{3}\right]^{2} N_{C}^{f} \frac{m_{\tilde{\chi}_{1}^{0}}^{5}}{m_{\tilde{f}}^{4}}
$$

where $\lambda$ is the general R-parity violating coupling, $m_{\tilde{f}}$ is the mass of the light sfermion interacting via the R-parity violating coupling, $N_{C}^{f}, Y_{f}$ are its color number and hypercharge, respectively, and a relevant gaugino component of the NLSP has been assumed. The R-parity violating two-body decay of a slepton into fermions reads

$$
\Gamma(\tilde{l} \rightarrow f f) \simeq \lambda^{2} \frac{m_{\tilde{l}}}{16 \pi}
$$

It is interesting to compare these decay rates with the rates of decay for low energy supersymmetry breaking models, Eq. (2), as a function of the coupling $\lambda$ and of the NLSP mass and the mass of the virtually exchanged sparticle. The comparison is striking. In the case of neutralinos as the NLSP, if photon events are to be seen in the presence of R-parity violating couplings, it follows from Eq. (8) that the coupling must be bounded by

$$
\lambda \lesssim 100\left(\frac{m_{\tilde{f}}}{\sqrt{F_{\mathrm{SUSY}}}}\right)^{2},
$$


which is a very stringent bound. Actually, for values of the sfermion masses, $m_{\tilde{f}} \simeq 300$ $\mathrm{GeV}$, and values of $\sqrt{F_{\text {SUSY }}} \simeq 10^{6} \mathrm{GeV}$, which are typical of low energy gauge mediated supersymmetry breaking models, the bound on the coupling is given by

$$
\lambda \lesssim 10^{-5} .
$$

This bound is of the order of magnitude of the strongest bounds on the trilinear R-parity violating couplings coming from flavor, lepton and baryon number violating processes [9]. Such low values of the couplings imply that R-parity violation is unlikely to be seen experimentally.

There is one exception to this general conclusion. This is the case in which the mainly gaugino-like neutralino is the NLSP and the dominant R-parity violating coupling is baryon violating, involving the third generation right handed top superfield, i.e. $\lambda_{3 i j}^{\prime \prime}$. In this case, assuming that the neutralino is lighter than the top quark, due to phase space constraints the decay of the lightest neutralino into three fermions will proceed via the small mixing between the stop and scharm eigenstates (which can be present at the tree level or appear via the $\mathrm{RG}$ evolution):

$$
\tilde{\chi}_{1}^{0} \rightarrow c j j
$$

In case the mixing is induced by radiative corrections, the additional loop-factors [10] will suppress the R-parity violating decay. Due to those extra supression factors the bounds on $\lambda^{\prime \prime}$ will be more than two orders of magnitude weaker than in Eq. (10). Even in this case, the bound is stringent, and hence, the observation of photons in the final state will still require small values of this R-parity violating coupling. Observe that an analogous suppression factor would not appear if $\lambda_{3 i j}^{\prime}$ were the dominant R-parity violating coupling, since in this case the neutralino can decay into left-handed bottoms without any phase space suppression.

In the case of sleptons as NLSP, the bound is even more striking,

$$
\lambda \lesssim\left(\frac{m_{\tilde{f}}}{\sqrt{F_{\mathrm{SUSY}}}}\right)^{2},
$$

meaning that the bound is typically two orders of magnitude smaller than in the case of neutralino as the lightest supersymmetric particle.

In the neutralino case, since all sfermions tend to couple to the NLSP, the bounds are quite general, on any possible $\lambda$. The strong bound on the couplings in the sfermion NLSP case, instead, only applies to the couplings of the lightest sfermion. Larger coupling of the heavier sfermions do not interfere with the possible signatures characteristic of the gauge mediated scenario. In general, assuming that the bound on the R-parity violating coupling of the NLSP, Eq. (13), is fulfilled, two possibilities may occur: i) The other R-parity violating couplings are sufficiently strong, so that two body R-parity violating decays of non-LSP sfermions can be observed; ii) Either the R-parity violating couplings for most of the sfermions are small and/or the neutralino is light enough so that the dominant decay mode for non-NLSP sfermions is R-parity conserving.

In case $i$ ) it would be easy to have independent experimental tests of R-parity violation and low energy supersymmetry breaking. In order to get a quantitative estimate 
of the values of the R-parity violating couplings for which this occurs, let us consider the R-parity conserving decay of a sfermion into a fermion and a neutralino:

$$
\tilde{f} \rightarrow f \tilde{\chi}_{1}^{0} ; \quad \tilde{\chi}_{1}^{0} \rightarrow l+N L S P \rightarrow l \bar{l} \tilde{G} .
$$

In the above, the lightest neutralino, $\tilde{\chi}_{1}^{0}$ may be real or virtual. If the neutralino is lighter than the sfermion, the sfermions may decay into neutralinos via a two-body decay channel, with a rate

$$
\Gamma\left(\tilde{f} \rightarrow f \tilde{\chi}_{1}^{0}\right) \simeq \frac{g^{2}}{8 \pi}\left(N_{12} T_{f}^{3}+\frac{Y_{f}}{2} \tan \theta_{W} N_{11}\right)^{2} m_{\tilde{f}}\left(1-\frac{m_{\tilde{\chi}_{1}^{0}}}{m_{\tilde{f}}}\right)^{2}
$$

where we have ignored terms proportional to the fermion Yukawa coupling. Due to the largeness of the weak gauge couplings, the R-parity violating decays are dominant only if

$$
\lambda>0.1
$$

unless cancellations between the different contributions to the effective sfermion-fermionneutralino coupling take place, or the neutralino mass is close to the sfermion mass. Such large R-parity violating couplings are subject to strong experimental constraints, and can only be realized in some particular cases [11].

Even if the neutralino were heavier than the sfermion, the dominance of the R-parity violating decay modes may still require fairly large couplings $\lambda$, due to the existence of three body R-parity conserving decay channels. For instance, let us consider the three body decay of a sfermion $\tilde{f}_{i}$ into another one, $\tilde{f}_{j}$, considered to be the NLSP. Assuming $m_{\tilde{\chi}_{1}^{0}}^{2}, m_{\tilde{f}_{i}}^{2} \gg m_{\tilde{f}_{j}}^{2}$, we get 12

$$
\Gamma\left(\tilde{f}_{i} \rightarrow f_{i} \bar{f}_{j} \tilde{f}_{j}\right) \simeq \frac{g^{4}}{48(2 \pi)^{3}} m_{\tilde{f}_{i}}^{3} \sum_{a, b=1}^{4} \frac{A_{a}^{i} A_{b}^{j}}{m_{\tilde{\chi}_{a}^{0}} m_{\tilde{\chi}_{b}^{0}}}
$$

and

$$
\Gamma\left(\tilde{f}_{i} \rightarrow f_{i} f_{j} \overline{\tilde{f}}_{j}\right) \simeq \frac{g^{4}}{192(2 \pi)^{3}} m_{\tilde{f}_{i}}^{5} \sum_{a, b=1}^{4} \frac{A_{a}^{i} A_{b}^{j}}{m_{\tilde{\chi}_{a}^{0}}^{2} m_{\tilde{\chi}_{b}^{0}}^{2}}
$$

where

$$
A_{a}^{i} \equiv\left(N_{a 1} \frac{Y_{f_{i}}}{2} \tan \theta_{W}+N_{a 2} T_{f_{i}}^{3}\right)
$$

and we are ignoring factors of order one and the phase space factors. We have implicitly assumed that $f_{i}$ and $f_{j}$ have opposite chiralities. Observe the different behaviour with the neutralino masses of the fermion conserving and fermion violating processes, which originates from a neutralino mass insertion in the first case. Due to the chirality flip, the fermion number conserving process scales like $\Gamma \simeq m_{\tilde{f}}^{3} / m_{\tilde{\chi}_{1}^{0}}^{2}$ while the fermion number violating one scales like $\Gamma \simeq m_{\tilde{f}}^{5} / m_{\tilde{\chi}_{1}^{0}}^{4}$ (If $f_{i}$ and $f_{j}$ had the same chirality, the opposite situation would occur). Since $m_{\tilde{f}}<m_{\tilde{\chi}_{1}^{0}}$, the fermion number conserving decay modes 
tend to be dominant. A lower bound on the $\lambda$ 's can be set in this case from the requirement that the two body $\mathrm{R}$-parity violating decays are dominant,

$$
\lambda \gtrsim \alpha_{w} \frac{m_{\tilde{f}}}{m_{\tilde{\chi}^{0}}} .
$$

This lower bound tends to be an order of magnitude lower than in the case of a real neutralino emission, discussed above. If the sfermion masses are close to each other, the lower bound on the R-parity violating coupling, Eq. (20) may be even smaller due to a possible strong phase space suppression [12].

In case $i$ ) the dominant decay mode for non-NLSP sfermions is R-parity conserving, overshadowing the R-parity violating decay modes. Still, even for values of the Rparity violating couplings $\lambda$ 's below the bounds, Eqs. (16) and (20), R-parity violating interactions may be detected via rare processes for a sizable range of values of those couplings.[9]

Until now, we have discussed the effects of trilinear couplings in the superpotential. What about R-parity violation by bilinears in the scalar potential and, when are they present? In principle, new effects may appear in this case, since the sneutrinos may acquire vacuum expectation values, inducing a mixing between the charged (neutral) leptons and the charginos (neutralinos) of the theory. The appearence of the bilinear terms in the scalar potential may be due to the following possibilities (we discuss here the scenario with explicit R-parity violation):

a) Only trilinear terms appear in the superpotential: In this case, although absent at tree level, bilinear terms in the scalar potential will be generated via the renormalization group evolution of the scalar mass parameters. Indeed, in the presence of Higgs and R-parity violating Yukawa terms, the bilinear soft supersymmetry breaking parameters are renormalized in a non-trivial way 13.

$$
\frac{d m_{H_{1} L}^{2}}{d t} \simeq \frac{h_{1 j k} \lambda_{L j k}}{16 \pi^{2}}\left(m_{j}^{2}+m_{k}^{2}+\frac{m_{H_{1}}^{2}+m_{L}^{2}}{2}\right),
$$

where $t=\ln \left(Q^{2}\right), m_{H_{1} L}^{2}$ is the off-diagonal Higgs slepton mass term appearing in the scalar potential, $h_{1 j k}$ are the Yukawa couplings of the trilinear term $H_{1} \Phi_{i} \Phi_{j}^{\prime}$ in the superpotential, while $\lambda_{L j k}$ is the R-parity violating coupling of the trilinear term $L \Phi_{i} \Phi_{j}^{\prime}$ in the superpotential. In the right hand side, we have only considered those terms proportional to the diagonal masses, which are presumably dominant and we have ignored the contributions governed by the soft supersymmetry breaking trilinear mass terms. The above means that in any theory in which trilinear couplings are present at the GUT scale, even in the absence of bilinear terms in the superpotential, bilinear soft supersymmetry breaking couplings will be generated at low energies.

Since the bilinear mass terms are only induced radiatively, with effects proportional to the small Yukawa couplings of the theory, we expect that the phenomenology will be still dominated by the trilinear couplings, and not by the effects of the small mixing. Neutrino masses are a possible exception to this rule, and they provide the most efficient constraints on the possible value of the sneutrino vacuum expectation values (see discussion below). One should stress, however, that if the trilinear R-parity violating couplings are allowed and the supersymmetric Higgs mass parameter $\mu \neq 0$, then there 
is no symmetry which can protect against tree level R-parity violating bilinear terms in the scalar potential and in the superpotential. Therefore, it is more realistic to consider case (b).

b) Both bilinear and trilinear terms appear in the superpotential: It is convenient to work in the basis in which the $\epsilon_{i} L_{i} H_{2}$ terms, Eq.(5), have been rotated away (for a basis independent approach see Refs. [14]) and to analyze the effects of the rotation. The new fields are then given by,

$$
H_{1}^{\prime}=\frac{\mu H_{1}+\epsilon L}{\sqrt{\mu^{2}+\epsilon^{2}}} \quad L^{\prime}=\frac{-\epsilon H_{1}+\mu L}{\sqrt{\mu^{2}+\epsilon^{2}}},
$$

where we have simplified the problem to the case of a single lepton superfield.

In the presence of soft supersymmetry breaking terms, after rotation of the Higgs and lepton superfields, these terms will generate not only additional trilinear couplings (see below), but also bilinear terms in the scalar potential [16], [17, 15, 18],

$$
V\left(H_{i}, \tilde{L}\right) \simeq\left(m_{H_{1}}^{2}-m_{L}^{2}\right) \sin \left(2 \theta_{L}\right) H_{1}^{\prime} \tilde{L}^{\prime}+\left(B_{H}-B_{L}\right) \mu \sin \theta_{L} H_{2} \tilde{L}^{\prime},
$$

where $\sin \theta_{L}=\epsilon / \sqrt{\mu^{2}+\epsilon^{2}}, m_{H_{1}}^{2}$ and $m_{L}^{2}$ are the scalar soft supersymmetry breaking parameters for the Higgs and slepton fields, respectively, while $B_{H}$ and $B_{L}$ are the soft supersymmetry breaking parameters governing the proportionality of bilinear terms in the potential and superpotential in the original basis. Eq.(23) shows the dependence of the bilinear R-parity violating terms in the scalar potential (generated by a bilinear term in the superpotential) at some scale $M$ on the soft terms at the same scale $M$. Since bilinear terms generically generate vacuum expectation values for sneutrinos, which in turn are strongly constrained by the limits on the neutrino masses, eq.(23) is a strong constraint on the combination of R-parity violating $\epsilon_{i}$ couplings and on the departure from universality of the soft supersymmetry breaking masses. A detailed numerical analysis of this constraint is beyond the scope of this paper. We observe only that in the presence of universal soft supersymmetry breaking couplings at some scale, the scalar potential has no bilinear R-parity breaking couplings at that scale. The kinetic terms for the Higgs and neutrino fields are a quadratic form and hence all what happens after the rotation is that we get a theory with new trilinear couplings $\bar{\lambda}_{i j k}$, with $\bar{\lambda}=\lambda$ for all couplings in which $L$ is not directly involved, while

$$
\bar{\lambda}_{L j k}=\lambda_{L j k}+h_{1 j k} \sin \theta_{L H}
$$

where $h_{1 j k}$ are the Yukawa couplings of the leptons and down quarks to the $H_{1}$ field. In this sense, in the presence of universal parameters at some large scale, there is no difference between a theory with only trilinear couplings, or a theory with both bilinear and trilinear couplings. Indeed, due to the non-renormalization theorem, once rotated away, there will be no regeneration of the $\epsilon$ term at low energies. In both cases, bilinear terms in the scalar potential will be generated radiatively at low energies.

The radiative generation of bilinear terms is the condition that one would like to achieve, in order to induce small neutrino masses, which, as happens with the neutralino mass matrix in the basis in which the $\epsilon$ parameters have been rotated away, 
are proportional to the sneutrino vacuum expectation values (the neutrino masses then still constrain the magnitude of the effective trilinear couplings and of the soft masses). This may happen in supergravity schemes, for exact universal boundary conditions. This exact universality, although possible, is not guaranteed by any symmetry in the theory. Another possibility is that the $\mu$ and $\epsilon$ terms are generated at scales larger than the scale leading to the superymmetry breaking parameters. In that case, assuming that the dynamics leading to supersymmetry breaking does not reintroduce them, the $\epsilon$ terms may be rotated away without worrying about the dangerous scalar terms in the superpotential.

A possibility, more appropriate within the context of this article is that supersymmetry is broken at scales much below the GUT scale, through a gauge mediated mechanism, which assures the universality of the Higgs and slepton soft supersymmetry breaking mass parameters at the scale $M$, at which the messengers are decoupled. Assuming that the dynamics leading to the appearence of the $\mu, \epsilon$ and $B$ terms does not modify the soft supersymmetry breaking scalar mass parameters, one can now rotate the $\epsilon$ parameter away, generating only small bilinear soft terms through the running between $M$ and $M_{Z}$. Observe that larger values of the R-parity violating couplings will be allowed in this case, due to the (assumed) small hierarchy of masses between $M$ and $M_{Z}$.

Finally, one can address the question of the relative magnitude of the two terms in Eq. (24). This is a model dependent question and in particular it is concievable that additional (e.g. U(1)) symmetries broken at high scale, distinguish between $L$ and $H_{1}$ [17], [19]. Both the bilinear and trilinear R-parity violating couplings are then realized as non-renormalizable operators and the latter can be strongly suppressed. Therefore, it is interesting to assume that there are regions where the effects of the non-vanishing sneutrino vacuum expectation values are the dominant ones and to see what kind of new phenomena they could imply. In particular, we would like to see the effects that can be obtained given the bounds on the $\nu$ masses.

Under the above assumption, the bilinear R-parity violating term in the scalar potential leads to a sneutrino vacuum expectation value and hence to new patterns in the mass matrices for neutralinos and charginos [20]. Those states become now mixed with neutrinos and charged leptons, respectively. In consequence, the structure of neutral and charged currents is modified and the couplings $\tilde{\chi}_{1}^{0} Z \nu$ and $\tilde{\chi}_{1}^{0} l W(\tilde{\nu} l l$ and $\tilde{l} \nu l)$ are generated at the tree level.

At the tree-level we now have

$$
\tilde{\chi}_{1}^{0} \rightarrow \nu Z \rightarrow \nu f \bar{f}
$$

with the width

$$
\Gamma\left(\tilde{\chi}_{1}^{0} \rightarrow \nu f \bar{f}\right) \simeq 2\left(\left(g_{V}^{f}\right)^{2}+\left(g_{A}^{f}\right)^{2}\right) \frac{G_{F}^{2} M_{\tilde{\chi}_{1}^{0}}^{5}\left(O_{\tilde{\chi}_{1}^{0} \nu}^{\text {“ }}\right)^{2}}{48 \pi^{3}} f(x),
$$

where $g_{V}^{f}, g_{A}^{f}$ are the vector and axial couplings of the fermion $f$ to the $Z$ gauge boson and

$$
O_{\tilde{\chi}_{1}^{0} \nu}^{\prime \prime}=\frac{1}{\sqrt{2}}\left[N_{13} N_{l 3}-N_{14} N_{l 4}+\sum_{i=5}^{7} N_{1 i} N_{l i}\right] \simeq g \frac{<\tilde{\nu}_{L}>}{M_{2}}
$$


where the 5th to 7 th components of the neutralino mass matrix in the basis of weak eigenstates are given by the neutrino fields, and a non-negligible gaugino component of the neutralino has been assumed. This coupling is suppressed by the mixing between the neutralino and the neutrino, which is controlled by the sneutrino vacuum expectation value, $\left\langle\tilde{\nu}_{L}\right\rangle$. The function $f(x)$ is a phase factor given by [20]

$$
f(x)=\frac{12}{x^{4}}\left[-\frac{x^{2}}{6}-\frac{1}{2}+\frac{1}{x^{2}}+\frac{1-x^{2}}{x^{4}} \ln \left(1-x^{2}\right)\right]
$$

with $x=M_{\tilde{\chi}_{1}^{0}} / M_{Z}$.

The neutralino can also decay into $W^{+}$and $l$, with a similar rate to the one given above [20],

$$
\frac{\Gamma\left(\tilde{\chi}_{1}^{0} \rightarrow f_{u} \bar{f}_{d} l\right)}{\Gamma\left(\tilde{\chi}_{1}^{0} \rightarrow \nu f \bar{f}\right)} \simeq \frac{K_{\tilde{\chi}_{1}^{0} l}^{2}}{8\left(\left(g_{V}^{f}\right)^{2}+\left(g_{A}^{f}\right)^{2}\right)\left(O_{\tilde{\chi}_{1}^{0} l}^{\prime \prime}\right)^{2}}
$$

with

$$
K_{\tilde{\chi}_{1}^{0} l}^{2} \simeq\left(U_{l 2} N_{13}+\sqrt{2} U_{l 1} N_{12}+\sum_{m=1}^{3} U_{l m+2} N_{1 m+4}\right)^{2}+\left(V_{l 2} N_{14}-\sqrt{2} V_{l 1} N_{12}\right)^{2}
$$

where the 3rd to 5 th components of the chargino mass matrices are given by the neutrino fields, and we have ignored the difference between the $Z$ and $W$ masses. It is easy to show that, in the presence of a non-neglibile gaugino component of the neutralino, $K_{\tilde{\chi}_{1}^{0} l} \simeq g<\tilde{\nu}_{L}>/ M_{2}$.

Comparing Eq. (2) with Eqs. (26) and (29), we conclude that the neutralino decay into the $\gamma \tilde{G}$ will be the dominant channel if

$$
\frac{<\tilde{\nu}_{L}>}{M_{2}} \lesssim \frac{10}{G_{F} F_{S U S Y}}
$$

For a characteristic value $\sqrt{F_{S U S Y}} \approx 10^{6} \mathrm{GeV}$, this bound is of order $10^{-6}$. Hence, unless the mixing angle between the neutrinos and neutralinos is extremely small, no photons will be observable in the presence of the R-parity violating decays induced by a sneutrino vacuum expectation value 2 . Note, that the bound, Eq.(31), would be even more stringent if a two body decay of the neutralinos into $Z \nu$ or $W l$ were open.

It is interesting to compare the above bounds, Eq. (31), with the experimental bounds coming from neutrino mass constraints. A quantitative estimate of the bounds on the couplings and mixing angles may be obtained by considering the neutrino mass generation in cases a) and b), and assuming that universal boundary conditions hold at some scale $\Lambda$. The minimization of the scalar potential leads to

$$
\frac{<\tilde{\nu}_{L}>}{\left\langle H_{1}\right\rangle} \approx \frac{m_{H_{1} L}^{2}}{m_{\tilde{\nu}_{L}}^{2}}
$$

${ }^{2}$ Observe that photons may be induced by the additional R-parity violating channel, $\tilde{\chi}_{1}^{0} \rightarrow \gamma \nu$, which is precisely the same signature as in gauge mediated models, with the gravitino replaced by the neutrino. This radiative generated decay rate is, however, subdominant compared to the three body decay channel 
where $m_{\tilde{\nu}_{L}}$ is the running sneutrino mass. Under the assumption of radiative generation of the bilinear terms, Eq. (32) can be rewritten as

$$
<\tilde{\nu}_{L}>\simeq \frac{\lambda_{L j k}\left\langle H_{1}\right\rangle h_{1 j k}}{16 \pi^{2}} \ln \left(\frac{\Lambda^{2}}{M_{Z}^{2}}\right),
$$

where we are ignoring factors of order one, proportional to the mass hierarchy of different sparticles (Higgs) at low energies [15]. From the neutralino mass matrix, it appears that the mass of the neutrinos is of order

$$
m_{\nu} \simeq g^{2} \frac{<\tilde{\nu}_{L}>^{2}}{M_{2}}
$$

where $M_{2}$ is a typical gaugino mass, of order of the weak scale. Hence, as a function of the experimental bound on the neutrino masses, $m_{\nu}^{\exp }$, strong constraints on the sneutrino vaccum expectation values, (and hence on the couplings and mixings) appear,

$$
g<\tilde{\nu}_{L}>\lesssim \sqrt{M_{2} m_{\nu}^{\exp }}
$$

For the three neutrinos we have [21]: $m_{\nu_{\tau}}^{\exp } \lesssim 24 \mathrm{MeV}, m_{\nu_{\mu}}^{\exp } \lesssim 170 \mathrm{KeV}$, and $m_{\nu_{e}}^{\exp } \lesssim$ $15 \mathrm{eV}$. For the second and third generations the bound becomes even stronger if the cosmological constraints coming from avoiding overclosing the universe 22] and valid for any generation, are considered,

$$
m_{\nu} \lesssim 40 \mathrm{eV}
$$

Therefore, the sneutrino vacuum expectation value is constrained to be below

$$
\frac{<\tilde{\nu}_{L}>}{M_{2}} \lesssim 10^{-5}, \quad \text { or, equivalently } \text { for } \quad M_{2} \simeq M_{Z} \quad<\tilde{\nu}_{L}>\lesssim 1 M e V .
$$

We see that the cosmological bounds on the sneutrino vacuum expectation value are close to the upper bound, Eq. (31), leaving only a small window for the R-parity violating decays to overshadow the decays into gravitinos. 3 Observe that, avoiding the above bound would require the existense of new, non-radiative decay modes of the heavy neutrinos, what would imply going beyond the physical framework under discussion [22]. For instance, the cosmological bounds may be avoided in the presence of Majorons, as

\footnotetext{
${ }^{3} \mathrm{~A}$ bound on the R-parity violating Yukawa couplings may also be obtained from Eqs. (33) and (37). Going to the basis in which the down quark and lepton masses are diagonal, the sneutrino vacuum expectation value becomes proportional to the square of those masses. Hence, a bound on the R-Parity violating coupling may be obtained from these considerations:

$$
\lambda_{L d d}^{2} \lesssim \frac{m_{\nu}^{e x p} M_{2}}{m_{d}^{2}\left(N_{C}^{d}\right)^{2}}\left(\frac{16 \pi^{2}}{\log \left(\Lambda^{2} / M_{Z}^{2}\right)}\right)^{2}
$$

where $m_{d}$ is the mass of the lepton or quark and $N_{C}^{d}$ is it number of colors. This means that the coupling $\lambda_{L b b}$ is heavily constrained by these considerations. Independently of the value of $\Lambda$, it follows $\lambda_{L b b} \lesssim 10^{-3}$. This bound is of the same order, but somewhat more general than the usual bound obtained from the low energy radiative corrections to neutrino masses [9], since it does not depend on the exact value of the left-right mixing mass parameter.
} 
those appearing in models of spontaneous breaking of R-parity [20], which we shall not discuss in this paper.

Other processes which can take place, via the neutralino-neutrino mixing are

$$
\tilde{\nu}_{L} \rightarrow l_{i} \bar{l}_{i} \quad \tilde{l} \rightarrow l_{i} \bar{\nu}_{i}
$$

with widths

$$
\Gamma \simeq\left(\frac{g^{2}<\tilde{\nu}_{L}>}{M_{2}}\right)^{2} \frac{m_{\tilde{\nu}}}{16 \pi} \quad \text { and } \quad \Gamma \simeq\left(\frac{g^{2}<\tilde{\nu}_{L}>}{M_{2}}\right)^{2} \frac{m_{\tilde{l}}}{16 \pi},
$$

respectively, where for simplicity we have ommitted factors of order one. The decay mode of sleptons and sneutrinos is induced by gauge coupling interactions. These processes will look similar to those induced via trilinear R-parity violating couplings. Hence, for the gravitino decay mode to be the dominant one, the bound on the sneutrino vacuum expectation value is defined by Eq. (13), with $\lambda$ replaced by $g^{2}\left\langle\tilde{\nu}_{L}\right\rangle / M_{2}$.

A final comment is in order. As said in the introduction, if the supersymmetry breaking scale is large, photons are not a characteristic signature of the minimal supersymmetric standard model, unless specific conditions are fulfilled. This can happen, for instance, when $M_{2} \simeq M_{1}$ and $\tan \beta \simeq 1$, in which case the two lightest eigenstates are a photino and a Higgsino [6]. For low values of $\mu$ one can get the Higgsino as the LSP, and the photino will have a one-loop induced decay into a Higgsino and a photon

$$
\tilde{\gamma} \rightarrow \gamma+\tilde{H}
$$

with a rate

$$
\Gamma \simeq \frac{G_{F} \alpha^{2}}{(4 \pi)^{3}}\left[\frac{m_{\tilde{\gamma}}^{2}-m_{\tilde{H}}^{2}}{m_{\tilde{\gamma}}}\right]^{3} G\left(m_{\tilde{\chi}^{+}}\right)
$$

where $G\left(m_{\tilde{\chi}^{+}}\right) \simeq \mathcal{O}\left(m_{W}^{2} / m_{\tilde{\chi}^{+}}^{2}\right)$. In general, the actual rate will be given by the above expression, but replacing $G\left(m_{\tilde{\chi}^{+}}\right)$by a complicated function of all particles contributing to the loop. In the above, we have assumed that the chargino- $W^{+}$contribution is the dominant one. The photon signatures arising from these models are easily distinguishable from the ones arising in low energy supersymmetry breaking models by the presence of massive final states carrying missing energy. In addition, the rate, Eq. (42), is not suppressed by any strong factor and hence, in case it becomes the relevant one, it will overshadow the R-parity violating decays of the neutralinos for values of the trilinear couplings $\lambda \lesssim 10^{-2}$.

We conclude that the observation of signatures with photons and missing energy, will put very strong limits on all the trilinear R-parity violating couplings appearing in the superpotential (or bilinear couplings in the cases that their effects are dominant compared to those induced by the trilinear couplings). The only exception to these rules can occur in the heavy gravitino case, for particular conditions in which the decay of photinos into a photon and Higgsinos is not suppressed. This decay process is easily distinguishable from the decay into LSP gravitinos. Relatively large R-parity violating couplings may still be present in this case. In the case of bilinear R-parity violating terms in the superpotential, we have obtained strong bounds on the sneutrino vacuum 
expectation values, and hence on the possible neutrino-neutralino or lepton chargino mixing coming from the constraints on the neutrino masses. If the cosmological bounds are considered, there is only a small window of allowed neutrino-neutralino (or lepton -chargino) mixings, which would allow the observation of mixing-induced R-parity violating decays in models of low energy supersymmetry breaking.

\section{ACKNOWLEDGEMENTS}

M.C. and C.W. would like to thank the members of the theory group at the University of Buenos Aires, Argentina, for their kind hospitality during the completion of this work. S.P. is partially supported by US-Poland Maria Sklodowska-Curie Joint Fund II (grant MEN/DEO-96-264) and by the Polish State Committee for Scientific Research under the grant 2 P03B 040 12. The authors would like to thank the Aspen Center for Physics, where most of this work has been done.

\section{References}

[1] P. Fayet, Phys. Lett. B70 (1977) 461; B86 (1979) 272; B175 (1986) 471.

[2] M. Dine, W. Fischler, and M. Srednicki, Nucl. Phys. B189 (1981) 575;

S. Dimopoulos and S. Raby, Nucl. Phys. B192 (1981) 353;

M. Dine and W. Fischler, Phys. Lett. B110 (1982) 227;

M. Dine and M. Srednicki, Nucl. Phys. B202 (1982) 238;

M. Dine and W. Fischler, Nucl. Phys. B204 (1982) 346;

L. Alvarez-Gaumé, M. Claudson, and M. Wise, Nucl. Phys. B207 (1982) 96;

C.R. Nappi and B.A. Ovrut, Phys. Lett. B113 (1982) 175;

S. Dimopoulos and S. Raby, Nucl. Phys. B219 (1983) 479.

[3] M. Dine and A.E. Nelson, Phys. Rev. D48 (1993) 1277;

M. Dine, A.E. Nelson, and Y. Shirman, Phys. Rev. D51 (1995) 1362;

M. Dine, A.E. Nelson, Y. Nir, and Y. Shirman, Phys. Rev. D53 (1996) 2658.

[4] S. Dimopoulos, M. Dine, S. Raby and S. Thomas, Phys. Rev. Lett. 76 (1996) 3494; S. Ambrosanio, G.L. Kane, G.D. Kribs, S.P. Martin and S. Mrenna, Phys. Rev. D54 (1996) 5395.

[5] H. Komatsu and J. Kubo, Phys. Lett. B157 (1985) 90; M. Quiros, G. Kane and H.E. Haber, Nucl. Phys. B273 (1996) 333; H.E. Haber and D. Wyler, Nucl. Phys. B323 (1989) 267; S. Ambrosanio and B. Mele, Phys. Rev. D55 (1997) 1399, Erratum Phys. Rev. D56 (1997) 3157.

[6] S. Ambrosanio, G.L. Kane, G.D. Kribs, and S.P. Martin, Phys. Rev. Lett. 76 (1996) 3498.

[7] L.J. Hall and M. Suzuki, Nucl. Phys. B231 (1984) 419; 
[8] S. Dawson, Nucl. Phys. B261 (1985) 297; V. Barger, G.F. Giudice, and T. Han, Phys. Rev. D40 (1989) 2987; H. Dreiner and G.G. Ross, Nucl. Phys. B365 (1991) 597.

[9] M. Hirsch, H.V. Klapdor-Kleingrothaus, and S.G. Kovalenko, Phys. Rev. Lett. 75 (1995) 17; K.S. Babu and R.N. Mohapatra, Phys. Rev. Lett. 75 (1995) 2276; K. Agashe and M. Graesser, Phys. Rev. D54 (1995) 4445; G. Battacharyya, J. Ellis and K. Sridhar, Mod. Phys. Lett. A10 (1995) 1583; G. Bhattacharyya, Nucl. Phys. Proc. Suppl. 52A (1997) 83; hep-ph/9709395; H. Dreiner, hep-ph/9707435;

[10] K.I. Hikasa and M. Kobayashi, Phys. Rev. D36 (1987) 724.

[11] G. Altarelli, J. Ellis, G.F. Giudice, S. Lola and M.L. Mangano, Nucl. Phys. B506 (1997) 3; D. Choudhury and S. Raychaudhuri, Phys. Lett. B401 (1997) 54; H. Dreiner and P. Morawitz, Nucl. Phys. B503 (1997) 55; J. Kalinowski, R. Rückl, H. Spiesberger and P. Zerwas, Z. Phys. C74 (1997) 595; K. Babu, C. Kolda, J. March-Russell and F. Wilczek, Phys. Lett. B402 (1997) 367; J. Hewett and T. Rizzo, Phys. Rev. D56 (1997) 5709; V. Barger, K. Cheung, K. Hagiwara and D. Zeppenfeld, Phys. Lett. B404 (1997) 147; G. Altarelli, G.F. Giudice and M.L. Mangano, Nucl. Phys. B506 (1997) 29; J. Ellis, S. Lola and K. Sridhar, Phys. Lett. B408 (1997) 252; T. Kon, T. Matsushita and T. Kobayashi, hep-ph/9707355; M. Carena, D. Choudhury, S. Raychaudhuri and C.E.M. Wagner, hep-ph/9707458, to appear in Phys. Lett B.

[12] M. Carena, G.F. Giudice, S. Lola and C.E.M. Wagner, Phys. Lett. B395 (1997) 225.

[13] B. de Carlos and P.L. White, Phys. Rev. D54 (1996) 3427 and references therein.

[14] S. Davidson and J. Ellis, Phys. Lett. B390 (1997) 210.

[15] E. Nardi, Phys. Rev. D55 (1997) 5772.

[16] R. Hempfling, Nucl. Phys. B478 (1996) 3.

[17] H.P. Nilles and N. Polonsky, Nucl. Phys. B484 (1997) 33.

[18] M.A. Diaz, J.C. Romao and J.W.F. Valle, preprint FTUV-97-32, hep-ph/9706315; M. Diaz, hepph/9711435; hepph/9712213.

[19] T. Banks, Y. Grossman, E. Nardi and Y. Nir, Phys. Rev. D52 (1995) 5319.

[20] M.C. Gonzalez-Garcia, J.C. Romao and J.W.F. Valle, Nucl. Phys. B391 (1993) 100; J.C. Romao et al., Nucl. Phys. B482 (1996) 3.

[21] Review of Particle Physics, Phys. Rev. D54 (1996) 1.

[22] For a recent review, see G.G. Raffelt, hepph/9712292. 